\title{
CONHECIMENTOS SOBRE PLANEAMENTO FAMILIAR EM ESTUDANTES DO ENSINO SUPERIOR
}

\author{
Paula Nelas \\ Adjunt Professor in Superior Health School \\ Polytechnic Institute of Viseu. PhD \\ pnelas@gmail.com \\ Emilia Coutinho \\ Adjunt Professor in Superior Health School \\ Polytechnic Institute of Viseu. PhD \\ Cláudia Chaves \\ Adjunt Professor in Superior Health School \\ Polytechnic Institute of Viseu. PhD \\ Odete Amaral \\ Adjunt Professor in Superior Health School \\ Polytechnic Institute of Viseu. PhD \\ Carla Cruz \\ Adjunt Professor in Superior Health School \\ Polytechnic Institute of Viseu. PhD
}

Fecha de Recepción: 9 Febrero 2018

Fecha de Admisión: 10 Abril 2018

\section{RESUMO}

Enquadramento: 0 planeamento familiar promove atividades que asseguram a saúde sexual e reprodutiva, garantindo informação promotora de uma vivência da sexualidade segura e saudável.

Objetivos: Determinar se as variáveis sociodemográficas, afetivas e sexuais influenciam os conhecimentos sobre planeamento familiar em estudantes do ensino superior.

Metodologia: Trata-se de um estudo quantitativo, transversal, descritivo e correlacional, com uma amostra de 199 estudantes do ensino superior, com uma média de idades de 20,89 anos ( $d p= \pm 2,01$ anos), maioritariamente do sexo feminino (76,4\%). 0 instrumento de colheita de dados foi o questionário que permitiu fazer a caracterização sociodemográfica, afetiva e sexual. Foi ainda incluída a Escala de Conhecimentos sobre Planeamento Familiar (Nelas, Silva, Ferreira, Duarte \& Chaves, 2010). Foram assegurados os procedimentos éticos. Os dados foram tratados com o programa SPSS (Statistical Package for the Social Sciences) versão 24 para Windows.

Resultados: Mais de metade $(86,9 \%)$ iniciou a vida sexual. A maioria que a iniciou $(78,6 \%)$ reporta aos $16-20$ anos. A maioria $(61,8 \%)$ não tem relação de namoro na atualidade, não tem relações sexuais no atual namoro 60,3\%. Usam contraceção 90,5\%. A maioria $(95,0 \%)$ utilizaria pre- 
servativo em relações ocasionais. Não vigiam a saúde sexual e reprodutiva $55,8 \%$ dos participantes e 0 enfermeiro esclarece dúvidas sobre saúde sexual e reprodutiva a $75,4 \%$ da amostra. Nunca realizou citologia cervical $63,1 \%$ das participantes. $56,8 \%$ não têm conhecimento sobre 0 planeamento familiar. As raparigas revelam mais conhecimento sobre planeamento familiar $(p=0,034)$. 0 rendimento mensal do agregado familiar tem relevância estatística, sendo os estudantes com maior rendimento que manifestam mais conhecimento $(p=0,047)$. Os estudantes da área da saúde possuem mais conhecimento $(p=0,001)$. Das variáveis afetivas e sexuais, apenas a vida sexual teve significância estatística, sendo os que ainda não iniciaram a ter mais conhecimento $(p=0,014)$.

Conclusão: Existe necessidade de intervenções promotoras da saúde sexual e reprodutiva, objetivando uma vivência da sexualidade saudável.

Palavras-chave: sexualidade; conhecimentos; planeamento familiar; estudantes; ensino superior

\section{ABSTRACT}

Knowledge about family planning in higher education students.

Background: Family planning promotes activities that ensure sexual and reproductive health, ensuring information that promotes a safe and healthy sexuality experience

Objectives: To determine if sociodemographic, affective and sexual variables influence the knowledge about family planning in higher education students

Methodology: This is a quantitative, cross-sectional, descriptive and correlational study with a sample of 199 higher education students, with a mean age of 20.89 years ( $\mathrm{sd}= \pm 2.01$ years), mostly female $(76.4 \%)$. The data collection instrument was a questionnaire that enabled the construction of the sociodemographic, affective and sexual characterization. The Family Planning Knowledge Scale (Nelas, Silva, Ferreira, Duarte \& Chaves, 2010) was also included. The ethical procedures were guaranteed. The data was processed with the SPSS program (Statistical Package for the Social Sciences) version 24 for Windows.

Results: More than half (86.9\%) have initiated their sexual life. The majority of these $(78.6 \%)$ reported the onset at $16-20$ years. Most of them (61.8\%) have no dating relationship at present, and $60.3 \%$. have no sex in the current relationship. $90.5 \%$ use contraception. The majority $(95.0 \%)$ would use condoms in occasional relationships. $55.8 \%$ of the participants do not monitor sexual and reproductive health and the nurse clarifies doubts about sexual and reproductive health with $75.4 \%$ of the sample. $63.1 \%$ of the participants never did a cervical cytology. $56.8 \%$ are not aware of family planning. Girls report more knowledge about family planning $(p=0.034)$. The monthly income of the household is statistically significant, with the highest income students showing more knowledge $(p=0.047)$. Health students have more knowledge $(p=0.001)$. Of the affective and sexual variables, only the sexual life had statistical significance, with those that had not initiated it, having more knowledge $(p=0.014)$.

Conclusion: There is a need for interventions to promote sexual and reproductive health, aiming at a healthy sexuality experience.

Keywords: sexuality; knowledge; family planning; astudents; higher education

\section{INTRODUÇÃO}

0 conceito de Saúde Sexual e de Saúde Reprodutiva consolidou-se a partir da segunda metade dos anos 90 do século XX, contribuindo para o incremento da importância da abordagem da educação sexual em contexto escolar, nomeadamente os conteúdos, metodologias a adotar e como implementar os projetos (Tavares, 2015). Uma vivência sexual segura e prazerosa resulta no bemestar emocional dos jovens, aos quais a saúde sexual e reprodutiva também diz respeito. Neste sen- 
tido, as atividades de Planeamento Familiar assumem-se como uma componente indispensável da prestação integrada de cuidados em saúde sexual e reprodutiva (Tavares, 2015).

A legislação Portuguesa contempla alguns fatores imprescindíveis em matéria de saúde sexual e reprodutiva dos jovens, garantindo 0 acesso a consultas de Planeamento Familiar a todos que a procuram, bem como a disponibilização dos métodos anticoncetivos, o acesso a consultas fora de área em qualquer serviço a qualquer jovem e 0 acesso livre à contraceção de emergência.

Atualmente existem várias consultas gratuitas, às quais os jovens podem recorrer para receber apoio na área da contraceção, planeamento familiar e sexualidade, nomeadamente a consulta de Planeamento Familiar nos cuidados de saúde primários, nos gabinetes de Saúde Juvenil do Instituto Português da Juventude e nas consultas para adolescentes, disponíveis em várias associações nãogovernamentais, freguesias e/ou Câmaras Municipais. Assim, é importante que os jovens conheçam as ofertas para que possam fazer a vigilância da sua saúde sexual e reprodutiva, através do acesso à informação, métodos de contraceção e serviços de saúde adequados (Andrews, 2016).

Num estudo desenvolvido por Reis e Matos (2007), que avalia os conhecimentos e atitudes de estudantes do ensino superior acerca dos métodos contracetivos, numa amostra constituída por 436 participantes, com idade compreendida entre os 18 e os 24 anos, ficou demonstrado que a maioria possui poucos conhecimentos acerca dos métodos contracetivos. Contudo, existe uma forte atitude preventiva face a uma gravidez indesejada. Encontraram-se diferenças estatisticamente significativas entre estudantes, tendo em conta o género, ou seja, as raparigas apresentam mais conhecimentos e preocupação preventiva face à contraceção. As mesmas autoras referem que se os jovens tiverem conhecimentos, informação e motivação acerca da contraceção, podem alterar as suas atitudes e, posteriormente, os seus comportamentos, compreendendo que a sexualidade pode ser vivida de forma saudável e feliz, necessitando simplesmente de ter uma atitude positiva, ou seja, utilizando a contraceção correta que os protejam de uma gravidez indesejada e de IST, as participantes do sexo feminino manifestam mais conhecimento e preocupação preventiva face aos risco e os participantes do sexo masculino mais aceitação do risco. Os resultados também indicam que a maioria é sexualmente ativo e utiliza simultaneamente preservativo e pílula. Os rapazes relatam mais frequentemente parceiros ocasionais e maior frequência de atividade sexual sob efeito de álcool ou drogas.

Apesar de os jovens terem fácil acesso à informação acerca da contraceção e comportamentos de risco, relacionadas à atividade sexual, esta informação nem sempre é a mais correta e nem se alicerça em fontes credíveis ou conhecimentos científicos. Neste sentido, a contraceção oral de emergência tem-se assumido como uma solução para evitar uma gravidez indesejável, mas sem se protegerem das IST's.

Tendo como ponto de partida estas premissas, a autora realizou uma investigação cujo objetivo era avaliar os conhecimentos e as atitudes dos jovens do ensino superior em relação à contraceção oral de emergência. Como resultados a maioria $(96,6 \%)$ relatou já ter ouvido falar da contraceção de emergência e sabiam que deve ser tomada após a relação sexual. Os meios de comunicação social e as consultas de planeamento familiar foram as fontes mais apontadas para obter o conhecimento da contraceção de emergência.

Relativamente aos conhecimentos dos jovens face à sexualidade, alguns estudos mostram que estes ainda revelam parcos conhecimentos sobre IST e sobre questões práticas referentes aos métodos contracetivos (Brestas, Ohara, Jardim \& Muroya, 2009; Vilar \& Ferreira, 2010).

0 estudo de Bitzer, Abalos, Apter, Martin \& Black (2016) analisou as barreiras associadas ao aconselhamento e atendimento eficazes de jovens relativas à contraceção e saúde sexual e reprodutiva. Foram identificadas barreiras como o preconceito sociocultural. 0 estudo de Ndayizigiye, 
Fawz, Livel e Ware (2017), cujo objetivo era explicar a adesão dos jovens a métodos anticoncecionais através da identificação de barreiras à sua utilização, verificou que existia uma maior aceitação associada ao número de profissionais de saúde envolvidos na consulta do planeamento familiar e com 0 número de diferentes tipos de contracetivos disponíveis. Foram identificadas as seguintes barreiras na adesão: falta de ajuste entre os métodos contracetivos disponíveis e os preferidos pelos jovens, medo na utilização de métodos contracetivos e falta de oferta de serviços de planeamento familiar. Os autores concluíram que onde os recursos de informação são escassos, a disponibilidade de métodos contracetivos por si só não garante a aceitação por parte dos jovens.

Para os jovens é fundamental integrar uma avaliação das necessidades de contraceção ao nível do planeamento familiar, já que a maioria tem relações sexuais pela primeira vez durante a adolescência. Os jovens não podem explicitamente solicitar aos profissionais de saúde os métodos contracetivos, sem que estes Ihes explicitem claramente qual o método mais indicado, é também fundamental ajuda-los a planear uma vida sexual saudável (Potter \& Santelli, 2015).

\section{METODOLOGIA}

0 estudo realizado é quantitativo, Iongitudinal, descritivo e correlacional, com uma amostra não probabilística por conveniência, sendo critério ser estudante do ensino superior. A amostra perfaz 199 participantes, 152 do sexo feminino e 47 do sexo masculino. A média de idade é de 20,89 anos $( \pm 2,01$ anos). Todos os procedimentos éticos e legais foram assegurados. Os dados foram tratados com recurso ao SPSS versão 24.0 para Windows.

0 instrumento de recolha de dados foi 0 questionário constituído por duas partes. A primeira caracteriza a amostra sociodemográficamente (sexo, idade, nacionalidade, residência e rendimento mensal do agregado familiar), a segunda parte permite a caracterização afetiva e sexual (início da vida sexual, relações sexuais no atual relacionamento, contraceção, tipo de contraceção, uso de contraceção de emergência, importância da utilização de preservativo para prevenção das IST's, utilização de preservativo, vigilância da saúde sexual e reprodutiva, acompanhante nas consultas de vigilância da saúde sexual e reprodutiva, realização de citologia cervical). Foi ainda incluída a Escala de Conhecimentos sobre Planeamento Familiar (Nelas, Silva, Ferreira, Duarte \& Chaves, 2010).

\section{RESULTADOS}

A amostra apresenta uma média de idades de 20,89 anos ( $d p=2,01$ anos, 0 sexo feminino representa $76,4 \%$ da totalidade da amostra. Residem em meio urbano 59,3\% dos participantes, e 0 rendimento mensal do agregado familiar mais elevado recai nos entre 1000-1499 euros mensais.

Mais de metade da amostra (86,9\%) já iniciou a vida sexual. Dos que já iniciaram a vida sexual, a grande maioria (78,6\%) refere ter iniciado entre os $16-20$ anos, $61,8 \%$ não namora na atualidade, $60,3 \%$ não têm relações sexuais no atual namoro, 90,5\% dos participantes faz contraceção, 95,0\% utilizaria preservativo se tivesse relações sexuais ocasionais. Em relação à contraceção usada, a percentagem mais elevada corresponde à pílula $(49,4 \%)$, seguindo-se $27,0 \%$ que referem outro tipo de contraceção e $21,3 \%$ usam o preservativo. Na amostra 63,5\% nunca fez contraceção de emergência e $36,5 \%$ admitem já ter feito.

Prevalecem os participantes que não vigiam a saúde sexual e reprodutiva $(55,8 \%)$. Dos que referem fazer vigilância da saúde sexual 44,9\% fazem-se acompanhar pelo(a) namorado(a). A maioria $(75,4 \%)$ admite que 0 enfermeiro de família esclarece dúvidas relativas à saúde sexual e reprodutiva. Quanto à regularidade com que os fazem vigilância da sua saúde sexual e reprodutiva, no total da amostra, estão em maioria os que dizem fazer anualmente $(77,3$. Importa ressalvar que todos os estudantes consideram importante a utilização de preservativo para prevenção das infecções de transmissão sexual. A maioria das participantes $(63,1 \%)$ nunca fez a citologia cervical, referindo $71,8 \%$ como motivo o desconhecimento. 
Na amostra, $80,2 \%$ das participantes do sexo feminino revelam conhecimentos sobre Planeamento Familiar. Verifica-se que no grupo de participantes com conhecimento sobre 0 Planeamento Familiar, a percentagem mais expressiva corresponde aos estudantes com idade compreendida entre os $20-22$ anos (46,5\%). Os resultados também revelam que os que residem em meio urbano possuem mais conhecimentos $(58,1 \%)$.

Os participantes que apresentam mais conhecimentos sobre Planeamento Familiar são os que já iniciaram a vida sexual $(80,2 \%)$, que não namoram na atualidade $(59,3 \%)$, que não têm relações sexuais na actual relação de namoro $(58,1 \%)$, que fazem contraceção $(89,0 \%)$, que se tivessem relações sexuais ocasionais utilizariam o preservativo (95,3\%), que não fazem vigilância de saúde sexual e reprodutiva $(55,8 \%)$, dos que vigiam, que o fazem anualmente $(81,6 \%)$ e que the são esclarecidas as dúvidas sobre saúde sexual e reprodutiva pelo enfermeiro de família $(86,8 \%)$.

As variáveis sociodemográficas sexo (feminino) e rendimento mensal do agregado familiar ( $\geq 1500$ euros) influenciam os conhecimentos sobre Planeamento Familiar. Relativamente às variáveis afectivas e sexuais apenas foi encontrada relação entre o facto de já ter iniciado a vida sexual e os conhecimentos sobre Planeamento Familiar.

\section{DISCUSSÂO DOS RESULTADOS}

Os resultados obtidos possibilitaram traçar um perfil sociodemográfico dos 199 estudantes do ensino superior, com predomínio de estudantes do sexo feminino (76,4\%). Quanto à caracterização afetiva e sexual, apurou-se que mais de metade da amostra (86,9\%) já iniciou a vida sexual $(86,2 \%$ sexo feminino vs. $89,4 \%$ sexo masculino). Dos que já iniciaram a vida sexual, a grande maioria $(78,6 \%)$ refere que aconteceu entre os $16-20$ anos (84,0\% sexo feminino vs. $61,9 \%$ sexo masculino). Sousa, Pimentel e Mata (2012), também verificaram que a maioria dos estudantes tinha iniciado a vida sexual (41,9\%) (38,1\% rapazes vs. $13,9 \%$ raparigas), com idade de início da vida sexual para as raparigas foi aos 16 anos e para os rapazes é aos 14,9 anos, o que é corroborado no presente estudo.

Constatou-se que a maioria não namora atualmente (61,8\%). Apurou-se que 60,3\% não têm relações sexuais no atual namoro, mas $39,7 \%$ admitem que $\operatorname{sim}(38,8 \%$ raparigas vs. $42,6 \%$ rapazes). Vilar e Ferreira (2010), verificou que quase metade dos jovens refere ter atualmente um relacionamento amoroso e que esse relacionamento abarca relações sexuais.

Outra questão importante refere-se ao facto de os estudantes usarem contraceção, constatando-se que quase a totalidade da amostra respondeu afirmativamente $(90,5 \%)$, com uma representatividade $92,6 \%$ para o sexo feminino e $83,3 \%$ para o sexo masculino. Perguntou-se se tivessem relações sexuais ocasionais utilizariam o preservativo, apurando-se que quase a totalidade da amostra $(95,0 \%)$ diz que utilizaria o preservativo $(98,0 \%$ sexo feminino vs $85,1 \%$ sexo masculino). No estudo de Vilar e ferreira (2010) ficou demonstrado que apenas uma pequena parte usa sempre 0 preservativo ou outro método contracetivo em simultâneo. Reis, M., Ramiro, L., Matos, M. G., \& Diniz, J. A., 2012), no que se refere às questões acerca do uso habitual dos métodos contracetivos, um número significativo de jovens referiu usar a pílula e o preservativo. Estas evidências empíricas corroboram as tendências encontradas no presente estudo. Em Portugal, 0 índice de jovens que usam os métodos contracetivos é elevado, sendo a pílula contracetiva utilizada por $85 \%$ das jovens (Matos, M. G., Reis, M., Ramiro, L., \& Equipa Aventura Social, 2011). De salientar que entre os jovens portugueses, de acordo com os mesmos autores, os métodos mais utilizados são o preservativo masculino e a pílula, o que foi corroborado com o presente estudo, uma vez que se verificou que a contraceção mais usada é a pílula $(49,4 \%)$, seguindo-se $27,0 \%$ que referem outro tipo de contraceção e $21,3 \%$ o preservativo. 
Constata-se que prevalecem os estudantes que não vigiam a sua saúde sexual e reprodutiva (55,8\%), com predomínio do sexo masculino (91,5\%), No total da amostra, 44,2\% referem que fazem vigilância, sendo a percentagem mais elevada no sexo feminino, com 55,3. Estes resultados reforçam a necessidade de se elucidar os jovens para a importância da vigilância da sua saúde sexual e reprodutiva. De acordo com a Lei n.. 120/99, de 11 de agosto, os jovens devem ter acesso à informação e educação sobre sexualidade e aos melhores serviços e apoios em matéria de saúde sexual e reprodutiva, incluindo 0 acesso à contraceção. Importa transmitir também aos jovens que, com a frequência da consulta de Planeamento Familiar, terão uma vida sexual satisfatória e segura (DGS, 2015). Neste sentido, os enfermeiros desempenham um papel importante na educação sexual e reprodutiva dos jovens no sentido de potenciar a sua capacidade de escolha de contraceção e sua utilização (Santos \& Figueiredo, 2015). Raquel, Tôrres, Cosme, Nascimento e Alchier (2013), no seu estudo sobre o papel do enfermeiro na saúde sexual e reprodutiva dos jovens da zona urbana do município de Pau dos Ferros, verificaram que os jovens relataram que, embora tenham sido bem acolhidos pelos enfermeiros e que os mesmos thes tenham esclarecido as sua dúvidas, não procuram como desejado os enfermeiros para falar sobre a sexualidade. Os mesmos autores sugerem que é de extrema importância que os enfermeiros debatam acerca de metodologias adequadas, educação em saúde, trabalho interdisciplinar, intersetorial e integral, vigilância à saúde, sexualidade, entre outros temas, para poderem trabalhar com os jovens.

Quanto ao uso da contraceção de emergência, apurou-se que mais de metade da amostra $(63,5 \%)$ refere nunca a ter feito, enquanto $36,5 \%$ admitem já a ter feito. Dos que já fizeram contraceção de emergência, $66,7 \%$, só a fez uma vez, sobressaindo também $22,2 \%$ que fizeram duas vezes. Morgado, Coutinho e Duarte (2013), no seu estudo sobre os significados outorgados pela jovem à vivência da sua sexualidade e sobre quais os significados que esta atribui à experiência de utilização de contraceção de emergência, numa amostra de 23 jovens do sexo feminino, constataram que as jovens expressaram uma grande "preocupação com as gravidezes indesejadas", todavia, "o uso inadequado de métodos contracetivos e o recurso a métodos pouco eficazes levam-nas ao uso da contraceção de emergência em situações extremas". Os mesmos autores, fazendo referência a Castro e Rodrigues (2009), referem que "a grande popularidade da contraceção de emergência reside, essencialmente, em tornar possível 0 anonimato - pela venda livre e auto-administração" (Morgado, Coutinho \& Duarte 2013). Todavia, ainda em conformidade com os autores citados, nem todos os jovens têm "à vontade para assumir a sua utilização, deixando transparecer sentimentos de vergonha e culpa em contextos de autoeficácia no comportamento contracetivo".

Constatou-se que a maioria das participantes $(63,1 \%)$ nunca realizou a citologia cervical, das quais a justificação dada pela maioria $(71,8 \%)$ foi 0 desconhecimento, enquanto $24,7 \%$ ainda não a fez por receio/medo e 3,5\% por vergonha. No estudo de Cruz e Jardim (2013), que procurou identificar o conhecimento de 118 estudantes sobre a citologia cervical e averiguar a prevalência da sua realização, constaram que este exame era conhecido por $54,2 \%$ das jovens, com conhecimento da sua finalidade por parte de $47,4 \%$ de jovens. Em 46,6\% das jovens sexualmente ativas, $65,4 \%$ já tinham realizado a citologia cervical, justificando a sua realização com a importância do exame para a sua saúde, tendo as restantes justificado a não realização por descuido $(14,5 \%)$ e por vergonha $(9,0 \%)$, como se constatou no presente estudo.

Constatou-se que todos os participantes consideram importante a utilização de preservativo para prevenção das IST's. Moreira, W. C., Lago, E. C., Viana, M. R. P., Carvalho, A. R. B., Frota, B. C., Pereira, P. S. L. (2015), realizaram um estudo que objetivou relatar a experiência de educação para a saúde vivenciada por estudantes do ensino superior, tendo observado a presença de baixo grau de conhecimento em relação aos métodos contracetivos e à prevenção de IST's, devido à 
ausência de orientações quer em contexto escolar, quer a nível familiar, o que os deixa ainda mais vulneráveis. Neste sentido, os mesmos autores consideram que este é um assunto que também deve ser discutido em contexto escolar, onde 0 enfermeiro assume um papel importante na educação para a saúde, promovendo a saúde sexual e reprodutiva.

Constatou-se que as participantes têm mais conhecimento sobre Planeamento Familiar, com diferença estatisticamente significativa $(p=0,034)$. Estes resultados estão em conformidade com os alcançados por Sousa, Pimentel e Mata (2012), cujo estudo demonstrou que são as raparigas que mais frequentam a Consulta de Planeamento Familiar.

Outra variável sociodemográfica com interferência estatística é o rendimento mensal do agregado familiar, tendo-se constatado que os participantes cujo agregado familiar tem maior rendimento mensal são os que manifestam mais conhecimento sobre o Planeamento Familiar ( $p=0,047)$, o que se poderá justificar com o facto de estes, devido à sua condição socioeconómica, poderem ter mais facilidade de acesso a informações e serviços de saúde.

0 facto de já terem iniciado a vida sexual teve relevância estatisticamente significativa, onde se salienta que os participantes que ainda não iniciaram a vida sexual apresentam mais conhecimento sobre 0 Planeamento Familiar ( $p=0,032)$. Estes resultados poder-se-ão explicar com 0 facto de, mesmo não tendo iniciado a vida sexual, já terem tido educação sobre a saúde sexual e reprodutiva no ensino secundário. Neste sentido, como menciona Tavares (2015), os serviços de saúde deverão dar resposta na área do planeamento familiar e da educação sexual e reprodutiva direcionada para os adolescentes.

\section{CONCLUSÕES}

Os resultados sugerem que se aposte mais em matéria de educação para a saúde sexual e reprodutiva dos jovens, procurando orientá-los para o facto de que esta se assume como um recurso que deve ser cuidado e preservado, desenvolvendo comportamentos de saúde salutares, que reúnam meios de luta contra as infecções de transmissão sexual e gravidezes indesejadas, potenciando uma vivência harmoniosa da sua sexualidade. É importante também que as instituições do Ensino Superior se transformem em Escolas Promotoras de Saúde, partindo-se do pressuposto que a sexualidade é uma das áreas contempladas no âmbito da Promoção da Saúde. Como tal, é fundamental a abordagem da educação sexual e reprodutiva em contexto de ensino superior.

No sentido de se facilitar e reforçar as aprendizagens na área da sexualidade e da saúde reprodutiva, assume-se também como importante que se transmita aos jovens que a consulta de Planeamento Familiar é um meio através do qual podem ter acesso à informação e ao aconselhamento sexual, prevenção e diagnóstico precoce das infecções de transmissão sexual, do cancro do colo do útero e da mama, métodos de contraceção e ainda o acesso gratuito ao contracetivo escoIhido.

\section{AGRADECIMENTOS}

Este trabalho é financiado por fundos nacionais através da FCT - Fundação para a Ciência e a Tecnologia, I.P., no âmbito do projeto UID/Multi/04016/2016. Agradecemos adicionalmente ao Instituto Politécnico de Viseu e ao Cl\&DETS pelo apoio prestado.

\section{BIBLIOGRAFIA}

Andrews, G. (2016). Contraceção: 0 que é que mudou na última década? Nursing, 153, 23-30.

Bitzer, J., Abalos, V., Apter, D., Martin, R., \& Black, A. (2016). Targeting factors for change: Contraceptive counselling and care of female adolescentes. The European Journal of Contraception \& Reproductive Health Care, 21(6), 417-430. Disponível em http://www.tandfon- 
line.com/doi/pdf/10.1080/13625187.2016.1237629?needAccess=true

Brêtas, J. R. S., Ohara, C. V. S., Jardim, D. P., \& Muroya, R. L. (2009). Conhecimento sobre DST/AIDS por estudantes adolescentes. Revista da Escola de Enfermagem da Universidade de São Paulo, 43(3), 551-557.

Castro, J. F., \& Rodrigues, V. M. C. P. (2009). Conhecimentos e atitudes dos jovens face à contracepção de emergência. Revista da Escola de Enfermagem da Universidade de São Paulo, 43(4), 8889-8994.

Cruz, D. E., \& Jardim, D. P. (2013). Adolescência e papanicolau: Conhecimento e prática. Adolescência \& Saúde, 10(Supl. 1 ), 34-42.

Portugal, Ministério da Saúde, Direção Geral da Saúde (2015). Plano Nacional de Saúde Escolar. Lisboa: DGS.

Matos, M. G., Reis, M., Ramiro, L., \& Equipa Aventura Social (2011). Saúde sexual e reprodutiva dos estudantes do ensino superior: Relatório do estudo - Dados nacionais 2010. Lisboa: FMH/UTL /CMDT/IHMT/UNL.

Moreira, W. C., Lago, E. C., Viana, M. R. P., Carvalho, A. R. B., Frota, B. C., Pereira, P. S. L. (2015).Sexualidade e prevenção de IST e HIV/AIDS entre idosos usuários da estratégia saúde da família. Revista Prevenção de infecção e Saúde. REPIS, 1(3). Acedido em http://www.ojs.ufpi.br/index.php/nupcis/article/view/3943

Morgado, A. P. S. F., Coutinho, E. C., \& Duarte, J. C. (2013). As jovens e a contraceção de emergência: Vivências da sexualidade. Millenium, 44, 141169.

Ndayizigiye, N., Smith Fawzi, M.C., Thompson Lively, C., \& Ware, N.C. (2017). Understanding low uptake of contraceptives in resource-limited settings: a mixed-methods study in rural Burundi. BMC Health Services Research, 17, 209. Doi: 10.1186/s12913-017-2144-0.

Nelas, P., Fernandes, C., Ferreira, M., Duarte, J., \& Chaves, C. (2010). Construção e validação da escala de conhecimentos sobre planeamento familiar. Sexualidade e educação sexual: Políticas educativas, investigação e práticas. Braga: Edições CIEd - Centro de investigação em Educação, Universidade do Minho.

Nelas, P. (2010). Educação sexual em contexto escolar: Uma intervenção contra a vulnerabilidade na vivência da sexualidade adolescente (Tese de doutoramento, Universidade de Aveiro). Acedido em http://ria.ua.pt/handle/10773/4656

Potter, J., \& Santelli, J.S. (2015). Contraceptive counseling for adolescents. Womens Health, 11(6), 737-741. Acedido em http://journals.sagepub.com/doi/pdf/10.2217/whe.15.75

Tôrres, T. R. F., Nascimento, E. G. C., Alchieri, J. C. (2013). 0 cuidado de enfermagem na saúde sexual e reprodutiva dos adolescentes. Adolescência \& Saúde, 10(Supl. 1), 16-26.

Reis, M., \& Matos, M. G. (2007). Contraceção: Conhecimentos e atitudes em jovens universitários. Psicologia, Saúde \& Doenças, 8 (2), 209-220.

Reis, M., Ramiro, L., Matos, M. G., \& Diniz, J. A. (2012). Os comportamentos sexuais dos universitários portugueses de ambos os sexos em 2010. Revista Portuguesa de Saúde Pública, 30(2), 105-114.

Santos, M. J., \& Figueiredo, A. (2015). Contraceção na adolescência: Aconselhamento e linhas de orientação. Revista Electrónica de Educação e Psicologia, 4, 62-75. Acedido em http://edupsi.utad.pt/index.php/component/content/article/80-revista3/123-contracecao-naadolescencia-aconselhamento-e-linhas-de-orientacao

Sousa, F., Pimentel, H., \& Mata, M. A. (2012). Concepção, opiniões e adesão dos adolescentes face às consultas de planeamento familiar. In: A. Lobo, A. Gonzalez, A. Carvalho, C. Rodrigues, C. Antunes, M. J. Monteiro, ... V. Rodrigues, Contextos de investigação em saúde (pp. 89-100). 
Chaves, UTAD, Vila Real: ESEVR. Acedido em http://www.esechaves.pt/documentos/ Contexto_livro.pdf

Tavares, G. M. (2015). Sexualidade na adolescência (Dissertação de mestrado, Instituto Politécnico de Portalegre). Acedido em https://comum.rcaap.pt/bitstream/10400.26/15002/1/ ESSTFC573.pdf

Vilar, D., \& Ferreira, P. M. (2010). A educação sexual dos jovens portugueses: Conhecimentos, fontes e impacto. In: Sexualidade e educação sexual: Políticas educativas, investigação e práticas (pp. 198-204). Braga: Universidade do Minho: Edições CIEd - Centro de Investigação em Educação. 
